\title{
Les entreprises irlandaises : nouveau moteur de la croissance économique nationale au lendemain de la crise?
}

Anne Groutel

\section{(e) OpenEdition \\ Journals}

\section{Édition électronique}

URL : http://journals.openedition.org/etudesirlandaises/4731

DOI : $10.4000 /$ etudesirlandaises. 4731

ISSN : 2259-8863

\section{Éditeur}

Presses universitaires de Rennes

\section{Édition imprimée}

Date de publication : 15 décembre 2015

Pagination : 31-44

ISBN : 978-2-7535-4366-9

ISSN : 0183-973X

\section{Référence électronique}

Anne Groutel, «Les entreprises irlandaises : nouveau moteur de la croissance économique nationale au lendemain de la crise? », Études irlandaises [En ligne], 40-2 | 2015, mis en ligne le 15 décembre 2017, consulté le 02 mai 2019. URL : http://journals.openedition.org/ etudesirlandaises/4731; DOI : 10.4000/etudesirlandaises.4731 


\title{
Les entreprises irlandaises : nouveau moteur de la croissance économique nationale au lendemain de la crise?
}

\author{
Anne Groutel \\ Université Paris 1 Panthéon-Sorbonne
}

Résumé

Éclipsées pendant des décennies par l'écrasante contribution des multinationales étrangères à l'économie, les entreprises irlandaises font, tout particulièrement depuis la crise, l'objet d'un regain d'attention de la part des autorités. Certains spécialistes prônent d'encourager l'entrepreneuriat pour lutter contre le chômage ou placent leurs espoirs dans ces entreprises qui s'internationalisent et pourraient bien stimuler la croissance économique nationale. Les conditions pour que les entreprises irlandaises en deviennent le prochain moteur sont-elles réunies?

Mots-clés : Politique industrielle, entrepreneuriat, entreprises, crise

\section{Abstract}

Dwarfed for decades by the overwhelming contribution of foreign multinationals to the economy, indigenous companies are now the focus of the Irish authorities' attention. Some experts call for nurturing entrepreneurship to fight unemployment and hope those Irish companies which internationalise will drive national growth. Are the conditions now prevailing in Ireland conducive to making Irish companies the next growth engine of the economy?

Keywords: Industrial policy, entrepreneurship, entreprises, crisis

Depuis la fin des années 1950, période où les gouvernants irlandais optèrent pour l'abandon du protectionnisme et la mise en œuvre d'une politique visant à attirer des entreprises étrangères à vocation exportatrice, le poids des multinationales dans l'économie irlandaise est allé en augmentant. Elles ont constitué le moteur de la croissance économique exceptionnelle que l'Irlande a connue du milieu des années 1990 au début des années 2000¹. D’ailleurs, Michael Casey, ancien économiste en chef à la Central Bank of Ireland et ancien membre du conseil d'administration du Fonds Monétaire International se fait fort de démystifier le rôle joué par les entreprises irlandaises dans ce succès : « Le tigre celtique n’a

1. Entre 1995 et 2000, l'Irlande a connu un taux moyen de croissance de $9 \%$ du produit national brut et de 9,8 \% du produit intérieur brut. Central Statistics Office, [http://www.cso.ie/en/media/csoie/releasespublications/ documents/economy/2001/nie_2001.pdf]. 
jamais existé, c'était un tigre américain². " Aujourd'hui les multinationales étrangères sont plus que jamais un pilier de l'économie irlandaise ${ }^{3}$.

Les entreprises irlandaises ${ }^{4}$ ont longtemps ${ }^{5}$ vécu dans l'ombre de ces géants qui étaient au cœur de la politique industrielle irlandaise $e^{6}$. Lattitude des autorités à leur égard ne commença à changer qu’à partir de la fin des années 2000, au plus fort de la crise financière et économique amorcée fin 2007. En 2008, Frank Ryan, alors directeur d'Enterprise Ireland ${ }^{8}$, faisait alors montre d'optimisme et se déclarait convaincu qu'après une première phase de développement économique généré par les investissements directs étrangers (IDE) ${ }^{9}$, et une seconde caractérisée par l'essor du secteur de la construction, la troisième serait marquée par la croissance des entreprises « indigènes ${ }^{10} »$. Les conditions sont-elles réunies pour que la vision de Frank Ryan devienne, à terme, réalité?

2. Michael Casey, «It was never a Celtic Tiger; it was an American one ", Ireland's Malaise, the Troubled Personality of the Irish Economy, Dublin, The Liffey Press, 2010, p. 82.

3. Les multinationales étrangères réalisent aujourd'hui $90 \%$ des exportations du pays. Forfás, Annual Business Survey of Economic Impact 2012, p. 4, [http://www.forfas.ielmedia/20032014-Annual_Business_Survey_of_Economic_Impact-Pulication.pdff, page consultée le 3 décembre 2014.

4. Il est parfois difficile de déterminer la nationalité d'une entreprise. « Une entreprise française, c'est quoi? », [http:// www.alliancegeostrategique.org/2010/04/19/une-entreprise-francaise-cest-quoi/, page consultée le $1^{\text {er }}$ décembre 2014. Tout au long de cet article, nous désignerons par les termes " entreprises irlandaises " ou " entreprises indigènes» (terme traduit littéralement), les sociétés dont les propriétaires soit résident en Irlande soit sont de nationalité irlandaise.

5. De grosses entreprises étrangères implantèrent des usines en Irlande à partir de la fin des années 1950, une époque charnière durant laquelle les dirigeants irlandais décidèrent d'abandonner la politique protectionniste en vigueur depuis 1932. Cependant, ce fut à partir du moment où le pays devint membre de la Communauté économique européenne, en 1973, que les multinationales américaines s'y installèrent en grand nombre.

6. Peadar Kirby, Celtic Tiger in Collapse, Explaining the Weaknesses of the Irish Model, Basingstoke, Palgrave Macmillan, 2010, p. 21-22, 39.

7. Pour de plus amples détails sur les raisons de la crise, voir : Anne Groutel, «L'Irlande : un avenir très incertain », Grande Europe, Focus, La Documentation française, mai 2011, [http://www.ladocumentationfrancaise.fr/pageseuropeld000428-irlande.un-avenir-tres-incertain-par-anne-groutel/article], page consultée le 16 décembre 2014; Valérie Peyronel, Catherine Coron et Régine Hollander (dir.), La crise financière et les économies du monde anglophone, Paris, Presses de la Sorbonne Nouvelle, 2010.

8. Enterprise Ireland est l'agence chargée principalement de l'aide au développement des entreprises irlandaises à fort potentiel de croissance et à vocation exportatrice.

9. Cette première phase de développement économique commença au milieu des années 1990. Cependant, à partir de 2003, le secteur de la construction devint le moteur principal de l'économie irlandaise. Cette seconde phase s’acheva avec l'éclatement de la bulle immobilière en 2008. Ce phénomène déclencha une crise financière sans précédant dans le pays.

10. John Walsh, «Export Hope », Business \& Finance, 18 juillet 2008. 


\section{Faire progresser l'économie irlandaise dans la chaîne de valeur mondiale à l'aube du nouveau millénaire : quel rôle pour le entreprises irlandaises?}

L'essor économique qu'a connu le pays à partir du milieu des années 1990 a certes permis d'élever le niveau de vie de la population irlandaise, mais il a, dans le même temps, entraîné la montée d'une spirale inflationniste. À mesure que les coûts augmentaient, l'Irlande perdait de sa compétitivité et de son attractivité pour les investisseurs étrangers. En 2004, le Enterprise Strategy Group, chargé par Mary Harney ${ }^{11}$ de préconiser une stratégie pour encourager la croissance et promouvoir l'emploi, estima que faire progresser l'économie dans la chaîne de valeur mondiale s'imposait pour faire face à la concurrence grandissante des pays d'Asie et parer aux risques de délocalisations des multinationales déjà installées en Irlande. L'objectif était désormais d'inciter les multinationales non seulement à fabriquer des produits à plus haute valeur ajoutée en Irlande mais également d'y mener des activités de recherche et développement. Faire de l'économie irlandaise une économie du savoir et promouvoir l'innovation devaient permettre à l'Irlande de rebondir.

Dans le cadre de son étude, ce groupe de réflexion se pencha sur le rôle que pouvaient jouer les entreprises irlandaises dans ce scénario. Son rapport révéla qu'à l'exception de quelques entreprises très dynamiques, piètres étaient les performances du secteur indigène dans son ensemble ${ }^{12}$.

En juillet 2005, Micheál Martin ${ }^{13}$ chargea donc le Small Business Forum ${ }^{14}$ de proposer des mesures concrètes afin de remédier aux obstacles auxquels les petites entreprises étaient confrontées ${ }^{15}$. L'objectif était de faire en sorte qu'elles puissent améliorer leur productivité et leurs performances en général. Elles pourraient alors croître et être en mesure d'exporter.

Le rapport du Forum contient une étude exhaustive des points faibles des entreprises irlandaises et des obstacles qui inhibaient leur croissance. Ses auteurs estimèrent qu'une action gouvernementale s'imposait car les entreprises irlandaises seraient appelées à jouer un rôle plus important dans l'économie irlandaise du

11. Marie Harney occupait le poste de Tánaiste et de ministre de l'Industrie, du commerce et de l'emploi.

12. Les entreprises indigènes étaient peu productives, peu innovantes, souvent par manque de moyens, et n'avaient pas ou peu recours aux nouvelles technologies. Seule une minorité d'entre elles exportaient, principalement vers le Royaume-Uni. Enterprise Strategy Group, Ahead of the Curve: Ireland's Place in the Global Economy, juillet 2004.

13. Micheál Martin était alors ministre du Commerce.

14. Le Small Business Forum fut crée en juillet 2005 par Micheál Martin. Ce groupe était composé d'entrepreneurs, de membres des organisations irlandaises représentant les petites entreprises, de personnels des agences publiques chargées du développement économique et d'universitaires. Il reçut pour mission de proposer des solutions pour améliorer les conditions dans lesquelles les petites entreprises opéraient en Irlande.

15. De fait, $97 \%$ des entreprises présentes en Irlande emploient moins de cinquante employés. 
futur ${ }^{16}$. Outre une batterie de mesures destinées à permettre aux entreprises de croître et de devenir plus innovantes, le Forum appela le gouvernement à élaborer une politique ciblée sur la promotion de l'entrepreneuriat, une politique qui avait jusqu'alors fait défaut ${ }^{17}$.

En septembre 2007, à la demande du gouvernement irlandais, Forfás ${ }^{18}$ publia "Towards Developing an Enterpreneurship Policy" ", un document qui devait servir de plan directeur pour l'élaboration de ladite politique. En premier lieu, ce document synthétisait les retombées positives de l'entrepreneuriat pour ou sur la croissance, la compétitivité et l'innovation mais également pour le développement régional et l'inclusion sociale. Il fixait un objectif ambitieux, celui de faire de l'Irlande l'une des nations les plus entrepreneuriales au monde :

L'Irlande se distinguera par sa forte culture entrepreneuriale, elle sera saluée pour ses entrepreneurs innovants et reconnue par les entrepreneurs comme étant l'un des meilleurs environnements au monde pour créer et développer une entreprise ${ }^{20}$.

Par ailleurs, certaines personnalités internationales du monde économique et politique exprimèrent publiquement leur scepticisme quant à la pérennité du modèle économique irlandais basé sur le " tout-multinationales ». Ainsi Robert Shapiro ${ }^{21}$ mit-il l'Irlande en garde. Celle-ci serait bien avisée de « se sevrer " de sa dépendance envers les investissements directs étrangers (IDE) qui, affirmait-il, ne doivent constituer qu'une étape dans le développement économique. Les Irlandais devaient selon lui, prendre leur destin en main et devenir une nation d'entrepreneurs. Le pays pourrait ainsi, à terme, disposer d'entreprises compétitives, têtes de file mondiales dans leurs secteurs. Venant de la part d'un conseiller de Barack Obama, ancien sous-secrétaire chargé du commerce et des affaires économiques, ces déclarations avaient de quoi interpeller les autorités irlandaises.

À ce coup de semonce, vinrent s'ajouter les critiques de Dan Breznitz ${ }^{22}$, expert international en matière de politique d'innovation. Il affirma sans détour que les

16. Small Business Forum, The Report of the Small Business Forum : Small Business Is Big Business, mai 2006, p. vIII. 17. Ibid., p. xvII.

18. Forfás est un organisme indépendant investi par l'État d'une mission de conseil en matière de développement industriel, de politique économique et d'innovation.

19. Forfás, Towards Developing an Enterprise Strategy, septembre 2007.

20. "Ireland will be characterized by a strong entrepreneurial culture, recognised for the innovative quality of its entrepreneurs, and acknowledged by entrepreneurs as a world-class environment in which to start and grow a business ". Ibid., p. 5.

21. Robert Shapiro était en visite en Irlande, en novembre 2008, pour participer à un séminaire sur les IDE organisé par la University College Dublin Business School. "Obama advisor says "Ireland must wean itself from dependence on FDI" - Foreign Direct Investment ", [http://www.finfacts.ielirishfinancenews/article_1015171. shtmll, page consultée le 8 décembre 2014.

22. Dan Breztniz, co-directeur du Innovation Policy Lab à la Munck School of Global Affairs de l'Université de Toronto, est l'auteur d'un ouvrage dans lequel il compare la politique d'innovation menée par Israël, Taiwan et 
efforts de l'État irlandais pour encourager la création d'entreprises innovantes à fort potentiel de croissance dans le secteur des nouvelles technologies n'étaient pas suffisants. Les autorités devaient, d'une part, encourager plus énergiquement toutes les entreprises irlandaises à se lancer dans la recherche et le développement et, d'autre part, opter pour des mesures qui limiteraient les risques financiers, inhérents à cette activité, pour les petites sociétés ${ }^{23}$.

Cependant, malgré ces appels à l'action, la politique de l'entrepreneuriat tant attendue ne fut pas élaborée par le gouvernement Fianna Fáil. En revanche, quelques mois plus tard il rendit public un projet ${ }^{24}$ plus global visant à stimuler l'innovation et dynamiser faire monter en gamme l'économie irlandaise. Force est de constater que si l'importance de développer l'entrepreneuriat y était reconnue, le rôle attribué aux entreprises irlandaises n'était donc qu'un rôle accessoire. Il ne s'agissait a priori pas d'en faire le prochain moteur de croissance mais plutôt qu'elles participent à la création d'un environnement milieu susceptible d'attirer les multinationales et les entrepreneurs étrangers :

Au cœur de l'économie intelligente, il y a un écosystème exemplaire en matière de recherche, d'innovation et de commercialisation. L'objectif est de faire de l'Irlande un pôle d'innovation et de commercialisation en Europe, un pays qui combine les caractéristiques d'une terre d'accueil attrayante pour les multinationales innovantes qui investissent lourdement dans la recherche et le développement tout en représentant un environnement d'incubation très attractif pour les meilleurs entrepreneurs en Europe et au-delà ${ }^{25}$.

En 2008, l'Irlande venait d'entrer en crise et ses dirigeants n'avaient pas encore, semble-t-il, pris la mesure de la gravité de la situation. Les autorités avaient jusqu'alors préparé la transition de l'économie irlandaise dans des conditions relativement favorables, sans qu'il n'y ait vraiment de sentiment d'urgence. Le pays jouissait d'une croissance économique stable ${ }^{26}$ et du taux de chômage le plus bas de la zone euro avec des taux annuels compris entre $4,4 \%$ et $4,6 \%{ }^{27}$.

l'Irlande. Innovation and the State: Political Choice and Strategies for Growth in Israel, Taiwan and Ireland, New Haven, Yale University Press, 2007.

23. Nicole Matthews, «Leading the Way to Success », Business \& Finance, 26 mars 2009.

24. Government of Ireland, Building Ireland's Smart Economy, A Framework for Sustainable Economic Renewal, Dublin, Stationery Office, 2008, [http://www.taoiseach.gov.ie/BuildingIrelandsSmartEconomy_1_.pdf], page consultée le 15 décembre 2014.

25. "The Smart Economy has, at its core, an exemplary research, innovation and commercialisation ecosystem. The objective is to make Ireland an innovation and commercialisation hub in Europe - a country that combines the features of an attractive home for innovative $R \dot{U} D$-intensive multinationals while also being a highly-attractive incubation environment for the best entrepreneurs in Europe and beyond. "Ibid., p. 8.

26. Dans les années précédant la crise financière de 2008, le taux de croissance annuel de l'Irlande se situait autour $5 \%$, [http://www.statistiques-mondiales.com/taux_de_croissance.htm], page consultée le 8 janvier 2014.

27. Statistiques mondiales, [http://www.statistiques-mondiales.com/ue_chomage.htm], page consultée le 8 janvier 2014. 
Mais, la crise allait changer complètement la donne et le début des années 2010 représenter véritablement une période charnière.

\section{Le tournant des années 2010}

Au début des années 2010, l'Irlande, après avoir été encensée pendant plus d'une décennie, était devenue l'un des maillons faibles de l'Union européenne. Outre les conséquences découlant directement de la crise dans le secteur bancaire, le gouvernement eut également à gérer la hausse spectaculaire du nombre de demandeurs d'emplois, qui passa à $14,5 \%$ en 2011. Remettre les finances du pays en bon ordre, faire en sorte que l'économie reparte sur des bases saines et lutter contre le chômage devinrent des priorités nationales. Ceci impliquait un retour aux fondamentaux c'est-à-dire promouvoir les exportations, moteur de croissance des années 1990.

Certes, les auteurs de Making it Happen, Growing Enterprise for Ireland, rapport publié par Forfás en 2010, réaffirment le rôle clé que jouent les multinationales dans la mesure où elles participent à l'implantation et au développement de nouveaux secteurs d'activité en Irlande tournés vers l'exportation et servent à la fois de caution et de vitrine à l'économie irlandaise ${ }^{28}$. Mais, ce document atteste dans le même temps de la volonté grandissante de cet organisme de faire prendre conscience aux gouvernants de l'urgence de promouvoir l'entrepreneuriat et de renforcer la contribution des entreprises nationales à l'économie :

La question de la trop grande dépendance de l'Irlande envers les multinationales étrangères et leurs exportations en particulier fait débat. En outre, la nature des activités menées en Irlande par les entreprises étrangères est en train de changer. Ces activités créent moins d'emplois. Étant donné le changement de dynamique qui est en train de s'opérer chez les entreprises nationales et la concurrence de plus en plus féroce à laquelle se livrent les pays pour attirer les investissements directs étrangers, l'heure est venue de prêter une attention nouvelle à l'optimisation du potentiel de croissance des entreprises indigènes. L'objectif global est d'augmenter la contribution des entreprises indigènes à la croissance future de l'Irlande ${ }^{29}$.

28. Forfás, Making it happen, Growing Enterprise for Ireland, septembre 2010, p. 79.

29. "At the same time, there is a contention that Ireland remains overly reliant on FDI in terms of its contribution to exports in particular. In addition, the nature of FDI being attracted to Ireland is changing, particularly in terms of its reduced employment intensity. Given the changing dynamic within indigenous firms, and in the context of an increasingly competitive global environment for the attraction of FDI, a renewed focus on accelerating the growth potential of the indigenous base is timely. ", Ibid. 
Il apparaît de plus en plus évident que s'en remettre aux multinationales étrangères ne peut suffire à résorber le chômage. Trouver des alternatives s'impose. Partant du constat que les entreprises créent le plus d'emplois dans les cinq premières années de leur existence, il est, selon ce rapport, impératif d'encourager la création d'entreprise. Promouvoir l'entrepreneuriat de croissance est aussi primordial afin que les entreprises irlandaises puissent accroître leurs exportations et ce d'au moins $33 \%$ (pour la période 2011-2015) ${ }^{30}$.

En 2012, Richard Bruton, ministre du Travail, des entreprises et de l'innovation au sein de la coalition associant le Fine Gael et le Labour Party, semblait avoir admis qu'un recadrage de la politique industrielle était inévitable :

Pour sortir de cette crise, nous devons nous efforcer de créer un moteur de croissance indigène. Même si les investissements directs étrangers en Irlande sont et resteront d'une importance cruciale, il est vital que nous trouvions la façon de permettre à nos entreprises indigènes d'améliorer leurs performances et d'exploiter au maximum les débouchés à l'export. Comme je l'ai dit précédemment, notre politique industrielle ne doit pas simplement avoir pour objectif d'attirer le prochain Google ou Microsoft en Irlande. Nous devons nous ingénier à créer le prochain Google ou Microsoft ici en Irlande ${ }^{31}$.

Un groupe de réflexion, le Entrepreneurship Forum, fut donc de nouveau chargé de formuler des propositions pour l'élaboration d'une stratégie ciblée sur l'entrepreneuriat. En effet, cette vision d'ensemble, exposant de façon claire ce qui devait être accompli et fixant des objectifs quantifiés, faisait cruellement défaut.

Par ailleurs, les conclusions du rapport d'évaluation, livré par Forfás ${ }^{32}$, des divers programmes gouvernementaux destinés à aider les entreprises nationales révèlent un certain nombre d'anomalies. En premier lieu, les agences chargées de la mise en œuvre des programmes destinés à développer l'entrepreneuriat avaient jusqu'alors fixé elles-mêmes les objectifs à atteindre, des objectifs qui manquaient parfois de clarté ${ }^{33}$. Dans ces conditions, estiment les rédacteurs du rapport de

\section{Ibid.}

31. "If we are to get out of this crisis, we must work hard to create an indigenous engine of growth. While multinational investment in Ireland is and will remain crucial, it is vital that we find ways of enabling our indigenous firms to perform better and take full advantage of export opportunities. As I have said before, our industrial policy must not just be aimed at attracting the next Google or Microsoft to Ireland - we must strive to create the next Google or Microsoft here in Ireland. " Richard Bruton cité dans un communiqué de presse d'Enterprise Ireland. Enterprise Ireland, "Call for applicants for next round of Enterprise Ireland's Competitive Start Fund ", press releases, 26 janvier 2012, [http://www.enterprise-ireland.com/en/News/PressReleases/2012-Press-Releases], page consultée le 15 janvier 2015.

32. Cette évaluation porte sur la période 2004-2010, Forfás, Forfás Evaluation of Enterprise Supports for Start-ups and Entrepreneurship, 14 avril 2014.

33. Ibid., p. 17. 
Forfás, il est difficile d'évaluer si le potentiel entrepreneurial a été exploité de façon optimale $^{34}$. Les auteurs de l'évaluation notent également des chevauchements entre les différents programmes. Enfin, comme l'avait déjà noté le Small Business Forum, la complexité de la répartition des responsabilités confiées à la multitude d'agences chargées de gérer ces programmes avait de quoi décourager le potentiel créateur d'entreprise ${ }^{35}$.

Malgré la pléthore de programmes destinés à aider à la création et à la croissance des entreprises, Dan Breznitz qui avait déjà porté un jugement sévère sur la politique gouvernementale irlandaise quelques années auparavant, ne se montra guère plus indulgent à l'égard de cette dernière en juin 2014 :

Les résultats de l'Irlande en matière de création d'entreprises indigènes innovantes et performantes sont médiocres, surtout dans le secteur technologique et sont à l'opposé du succès qu'elle remporte dans la promotion des investissements directs étrangers. Attirer des multinationales est positif et a permis de créer beaucoup d'emplois en Irlande [...] Mais la fragilité du secteur des entreprises indigènes est alarmante ${ }^{36}$.

\section{Irlandais : levez-vous et entreprenez!}

À l'analyse, le rapport élaboré par le Entrepreneurship Forum reprend les thèmes déjà présents dans le rapport du Small Business Forum comme par exemple la nécessité de développer le réseautage et le mentorat d'entreprise. En 2006, il était déjà question de promouvoir la création d'entreprise particulièrement auprès des femmes et de la communauté immigrée. Désormais, les demandeurs d'emploi, les seniors et les jeunes, diplômés ou non, font également partie de ceux qu'il faut pousser à s'engager dans cette voie.

Le rapport du Entrepreneurship Forum se démarque toutefois sensiblement de l'approche de ses prédécesseurs dans la mesure où il préconise que l'État limite son rôle à celui de "facilitateur ", et s'abstienne de mettre en œuvre de nouvelles initiatives privilégiant l'amélioration de programmes déjà mis en œuvre. Veiller à ce que les entreprises puissent obtenir aisément les financements dont elles ont besoin, faciliter l'accès à l'information, mettre en place un régime fiscal incitatif, faire en sorte que le système éducatif inculque l'esprit d'entreprise à tous les niveaux et que le système de protection sociale ne désavantage pas les créateurs

34. Ibid., p. 17-18.

35. Ibid. p. 18.

36. "Ireland has done very poorly on creating innovative and successful indigenous companies, especially in the technology sector, as opposed to being very successful at attracting in multinationals. Attracting foreign direct investment is also positive and has supplied many Irish jobs [...] But Ireland's indigenous sector is alarmingly weak. " "Ireland could learn the lessons of social entrepreneurship ", The Irish Times, 9 juin 2014. 
d'entreprises, telles sont les tâches auxquelles l'État doit s'atteler. Car il revient aux citoyens en général et aux entrepreneurs de prendre en main leur destin :

L'entrepreneuriat en Irlande commence avec une culture de citoyenneté engagée $[. .$.$] Des citoyens engagés sont responsables de leurs réus-$ sites et de leurs échecs, et s'engagent à en tirer les leçons [...] Une Irlande vraiment entrepreneuriale dépend d'entrepreneurs solides et autonomes, qui sont dévoués à leurs clients, leurs équipes et leurs réseaux. Les entrepreneurs doivent se tourner plus vers leurs pairs et attendre moins du soutien du gouvernement pour atteindre leur objectif ${ }^{37}$.

Plus d'autonomie pour les entrepreneurs et d'entraide, un discours quelque peu inhabituel en Irlande mais qui s'explique peut-être par le fait que le Forum ait été présidé par Sean O'Sullivan ${ }^{38}$, un célèbre entrepreneur touche-à-tout, américain d'origine irlandaise, revenu vivre " au pays ».

\section{圈 Aperçu de l'action gouvernementale destinée à aider la création et la croissance des entreprises irlandaises}

Une petite phrase d'introduction : "Il semblerait que le gouvernement irlandais ait bien pris acte de certaines des recommandations émises dans les rapports précédemment cités. "

En 2014, le gouvernement a enfin accédé à une demande récurrente des entreprises, à savoir, simplifier le parcours des créateurs d'entreprise et des entrepreneurs. Dans le cadre de son programme, Action Plan for Jobs 2014, le ministère du Travail a créé les Local Enterprise Offices (LEOs) en avril 2014, des guichets uniques désormais chargés de renseigner et d'aider les petites et très petites entreprises en offrant subventions et formation ${ }^{39}$.

Le gouvernement a également publié sa stratégie pour promouvoir l'entrepreneuriat ${ }^{40}$. Ce document constitue en soi un pas en avant. Les deux avant-propos, l'un du Premier ministre l'autre du ministre du Travail, sont de bon augure puisque l'importance de l'entrepreneuriat y est clairement soulignée tout comme l'est l'objectif de faire de l'Irlande l'un des pays les plus dynamiques au monde

37. «Entrepreneurship in Ireland starts with a culture of engaged citizenship [...]. Engaged citizens take responsibility for their own progress and failure, and commit themselves to learning [...]. A truly entrepreneurial Ireland is dependent on strong and self-reliant entrepreneurs, who are focused on their customers, their teams and their networks. Entrepreneurs must look more toward their peers and less to government support to achieve their goal. » Entrepreneurship Forum, Strengthening the Start-up Community, Report of the Enterpreneurship Forum, janvier 2014, p. 5.

38. Sean O'Sullivan est tout à la fois un entrepreneur en série, un inventeur et un investisseur. [http://www.rte.ie/ tv/dragonsden/profile_seanosullivan.html].

39. Department of Jobs, Enterprise and Innovation, Action Plan for Jobs 2014, février 2014, p. 18.

40. Department of Jobs, Enterprise and Innovation, National Policy Statement on Entrepreneurship in Ireland, septembre 2014. 
en matière d'entrepreneuriat. Le gouvernement irlandais estime qu'il serait ainsi possible de créer 93000 emplois d'ici à 2019 ${ }^{41}$. Mais pour atteindre cet objectif les créations d'entreprises devront augmenter de $25 \%$ (plus 3000) par an. Il devra en aller de même pour le taux de survie des entreprises de moins de cinq ans (plus 1800 par an). Enfin, le potentiel de développement des entreprises, une notion quelque peu vague, devra aussi augmenter de $25 \%{ }^{42}$.

Cependant, force est de constater que le reste de ce document tant attendu est assez décevant. En guise de stratégie, le ministère du Travail a, en réalité, produit une synthèse contenant les différentes mesures qui existent déjà pour encourager la création d'entreprise et, hormis ceux qui viennent d'être cités, les objectifs ne sont pas quantifiés.

L'approche prônée par le Entrepreneurship Forum a été adoptée. Il est donc clairement spécifié dans le rapport que les autorités irlandaises comptent laisser les rennes aux entrepreneurs potentiels et aux chefs d'entreprise ${ }^{43}$. Le gouvernement veillera à ce que les programmes scolaires et universitaires intègrent la promotion de l'esprit d'entreprise afin qu'à terme la culture irlandaise en soit imprégnée $e^{44}$. Mais globalement l'action des autorités se cantonnera à instaurer l'environnement le plus favorable possible à l'entrepreneuriat, entre autres, par le biais de la politique fiscale $e^{45}$, de la simplification des procédures administratives et du droit des sociétés ${ }^{46}$.

L'autre grand défi réside dans la nécessité d'aider les entreprises existantes à se développer suffisamment pour qu'elles soient en mesure d'exporter. Aussi le ministère des Affaires étrangères et du Commerce a-t-il redoublé d'efforts pour organiser des missions de prospection commerciale à l'étranger afin d'aider les entreprises irlandaises à trouver de nouveaux débouchés en particulier dans les pays émergents et en Chine ${ }^{47}$.

Toutefois, le récent sondage mené auprès des entreprises exportatrices irlandaises par l'Irish Exporters Association ${ }^{48}$ est édifiant. En premier lieu, elles estiment que le soutien de l'État est soit moyen, (44,3\%) insuffisant (18,6\%) ou inexistant $(9,8 \%)^{49}$. Cela s'explique peut-être par le fait que la moitié des sondés ne connaissait aucun des programmes destinés à aider les entreprises. Qui plus est,

41. Charlie Taylor, "Government outlines plans to create 93,000 new jobs », The Irish Times, 9 octobre 2014.

42. Department of Jobs, Enterprise and Innovation, National Policy Statement on Entrepreneurship in Ireland, op. cit., p. 6.

43. Department of Jobs, Enterprise and Innovation, National Policy Statement on Entrepreneurship in Ireland, op. cit., p. 8.

44. Ibid., p. 20-23.

45. Ibid., p. 24-27.

46. Ibid., p. 28-29.

47. Houses of Oireachtas, Dáil Éireann, Joint Committee on Jobs, Enterprise and Innovation, 15 juillet 2014.

48. Irish Exporters Association, Export Ireland Survey and International Trade Finance Review 2014.

49. Ibid., p. 74. 
$68,9 \%$ de ceux qui ont eu accès aux informations sur ces programmes déclarent avoir été mal informés ${ }^{50}$. Enfin, $65,5 \%$ se plaignent que les lourdeurs administratives entravent la bonne marche de leur entreprise ${ }^{51}$.

Malgré cela, les entreprises irlandaises se développent et montrent des signes encourageants qui pointent vers une reprise de l'économie nationale.

\section{La montée en puissance des entreprises irlandaises}

En premier lieu, on estime à une vingtaine le nombre d'entreprises irlandaises qui sont parvenues à se développer et à accéder au rang de multinationales ${ }^{52}$. Certes, pour le moment aucune d'entre elles n'appartient au secteur de la haute technologie, secteur phare du dynamisme économique, mais cette évolution est un phénomène très positif qui marque une maturité grandissante de l'économie irlandaise $\mathrm{e}^{53}$. Les investissements directs sortants ${ }^{54}$, qui avaient augmenté de façon spectaculaire (plus $325 \%$ entre 2000 et 2005), ont fait preuve d'une grande résilience face à la crise ${ }^{55}$. Ceci est d'autant plus important que des études ont montré l'impact positif de ces derniers sur l'emploi en Irlande ${ }^{56}$. Aussi les autorités irlandaises encouragent-elles cette tendance ${ }^{57}$.

Par ailleurs, les entreprises irlandaises réalisent aujourd'hui une plus grande part de leurs ventes à l'export. De $37 \%$ en 2003, elles sont passées à $52 \%$ en 2012. Même si les produits manufacturés issus de l'industrie agro-alimentaire représentent toujours une part substantielle de ces exportations (41\%), on note une augmentation importante de la part des services informatiques, de communication et d'information (plus $20 \%$ par rapport à 2003) (5) $^{58}$ Ce qui tend à montrer que l'économie irlandaise est progressivement en train de progresser dans l'échelle de valeur mondiale de monter en gamme. Les entreprises technologiques irlandaises sont actuellement au nombre de 806, un nombre qui continue d'augmenter. Qui plus est, une cinquantaine d'entre elles ont traversé l'Atlantique pour s'implanter dans la Silicon Valley. Certaines louent des bureaux au Enterprise

50. Ibid., p. 75.

51. Ibid., p. 76.

52. Noelle Donnelly, "The Emergence and Internationalization of Irish MNEs ", International Studies of Management and Organization, vol. 43, no. 1, printemps 2013, p. 26-51.

53. Louis Brennan et Rakhi Verma, "Outward FDI in Ireland and its Policy Context ", Columbia FDI profiles, Vale Columbia Center on Sustainable International Development, 12 février 2013, [http://academiccommons. columbia.edu/catalog/ac\%3A158995], page consultée le 11 janvier 2015.

54. Les investissements directs sortants sont les investissements des entreprises irlandaises à l'étranger.

55. Excepté en 2011, année marquée par une chute brutale, les investissements directs sortants des entreprises irlandaises ont bien résisté et sont aujourd'hui de nouveau en phase de croissance. Ils s'élevaient à presque 23 milliards de dollars en 2013. Unctad, World Investment Report 2014, p. 205.

56. Louis Brennan et Rakhi Verma, op. cit., p. 6.

57. Ibid., p. 5.

58. Forfás, Business Survey of Economic Impact 2012, mars 2014, p. 14-15. 
Ireland Centre et bénéficient du soutien du Irish Technology Leadership Group, un groupe constitué de membres influents de la diaspora irlandaise très bien introduits dans les milieux d'affaires de la Silicon Valley ${ }^{59}$.

Brennan et Verma ${ }^{60}$ sont très optimistes quant au potentiel des entreprises irlandaises et aux chances de les voir véritablement stimuler la croissance nationale. D'ailleurs, Julie Sinnamon, directrice d'Enterprise Ireland, affirmait récemment que les exportations des entreprises irlandaises n'ont jamais été aussi élevées $^{61}$. Il convient cependant de mettre ces signes encourageants en perspective. Certes, l'impact des entreprises irlandaises sur l'économie nationale ${ }^{62}$ est égal, si ce n'est légèrement supérieurn, à celui des multinationales, mais ces dernières représentent toujours $90 \%$ des exportations du pays.

\section{L'Irlande : une nation d'entrepreneurs?}

Un certain nombre d'entreprises irlandaises se développent et s'internationalisent. Mais sommes-nous pour autant en présence d'une nation d'entrepreneurs? En 2008, la population se caractérisait par un esprit entrepreneurial bien supérieur à la moyenne européenne selon le Global Entrepreneurship Monitor (GEM) Report ${ }^{63}$. Mais la crise a eu un effet dévastateur sur la création d'entreprises en Irlande. Alors qu'en 2006, 16700 nouvelles sociétés avaient été créés, ce nombre n'était plus que de 11200 en 2010. L'Irlande, l'un des pays d'Europe les plus dynamiques en matière de création d'entreprises avant la crise, est brusquement devenue l'un des plus pusillanimes. Une prudence somme toute justifiée quand on sait que des entreprises créées en 2007 , seules $48,4 \%$ avaient survécu en $2012^{64}$.

Il est à craindre que le traumatisme engendré par la crise n'ait inhibé l'esprit d'entreprise en Irlande. En effet, contrairement aux conclusions optimistes du rapport de 2008, le Global Entrepreneurship Development Index 2014 (GEDI), montre clairement que l'Irlande est un pays très frileux à l'égard du risque ${ }^{65}$. D'aucuns affirment que ce serait un trait caractéristique de sa population et qui serait

59. Barbara McCarthy, "The successful entrepreneurs who have moved to Silicon Valley and are taking on the tech world ", The Irish Independent, 31 août 2014.

60. Louis Brennan et Rakhi Verma, op. cit., p. 6.

61. Houses of Oireachtas, Dáil Éireann, Joint Committee on Jobs, Enterprise and Innovation, 15 juillet 2014.

62. Prenant en compte les salaires versés et les achats de matières premières et de services, Julie Sinnamon estime cet impact à 18 milliards d'euros.

63. En 2008, le taux de création d'entreprises était de 4,3\% alors que la moyenne européenne n'était que de 2,7 \%. Paula Fiztsimmons et Colm O'Gorman, Entrepreneurship in Ireland, Global Entrepreneurship Monitor, The Irish Annual Report, 2008.

64. Department of Jobs, Enterprise and Innovation, National Policy Statement on Entrepreneurship in Ireland, op. cit., p. 14.

65. Ibid., p. 17. 
doublé d'une tendance au défaitisme. Interrogé par les membres du Comité parlementaire mixte sur le travail, l'entreprise et l'innovation, lors de la présentation du rapport du Entrepreneurship Forum, Sean O'Sullivan lui-même reconnaissait que " regarder dans la mauvaise direction et regarder vers l'échec est une façon d'aller à l'échec. Nous faisons beaucoup cela, cela fait partie de notre culture ${ }^{66}$ ". Et le président du Comité d'ajouter : "Il y a une culture de la non-prise de risque. Les parents encouragent leurs enfants à choisir un emploi sûr comme comptable ou avocat ou quelque chose du même genre ${ }^{67}$. " Michael Casey partage cette opinion dans le portrait sans concession qu'il dresse de ses concitoyens ${ }^{68}$. Pourtant, faute de recherche suffisante en la matière, il n'existe aucune certitude quant à l'influence exacte de la culture sur l'entrepreneuriat ${ }^{69}$.

D'ailleurs, un climat économique, national et mondial, favorable peut se révéler être l'élément qui va déclencher le passage à l'action. La période de croissance et de prospérité que connut l'Irlande pendant un peu plus d'une décennie contribua certainement à engendrer cette forte propension à créer des entreprises. On note d'ailleurs une reprise sensible de la création d'entreprises depuis un an, signe que la confiance en l'économie nationale est en train de revenir. Deux études, l'une menée par la Irish Exporters Association ${ }^{70}$, l'autre par la Irish Software Association ${ }^{71}$, confirment une tendance à l'optimisme.

Des exportations en hausse, un retour de la confiance, des créations d'entreprises en augmentation, certaines conditions semblent réunies pour que les entreprises irlandaises contribuent dans une plus large mesure à l'économie nationale. Il est néanmoins difficile d'imaginer qu'elles puissent devenir le prochain moteur de la croissance dans un avenir proche. D'ailleurs même si le discours des gouvernants irlandais a indiscutablement changé, ils semblent douter que les entreprises irlandaises puissent former à elles seules une masse critique, un écosystème, puisqu'ils continuent de chercher à attirer des entrepreneurs étrangers. Et de s'interroger sur le rôle de l'État en tant que simple "facilitateur ». Est-ce vraiment

66. "Looking in the wrong direction and looking towards failure is a way to get towards failure. We do that a lot as part of our culture. " Houses of Oireachtas, Dáil Éireann, Joint Committee on Jobs, Enterprise and Innovation, 23 février 2014.

67. "There is a culture of not taking a risk. Parents encourage their children to get a secure job as an accountant or solicitor or whatever. "Ibid.

68. "The evidence, such as it is, suggests that the Irish gene pool does not produce entrepreneurs in great numbers and that our culture does not nurture them. " Michael Casey, op. cit., p. 67.

69. Erkki Audio, Pathak Saurav et Karl Wennberg, "Consequences of Cultural Practices for Entrepreneurial Behaviors ", Journal of International Business Studies, vol. 44, no. 4, p. 334-362.

$70.71 \%$ des sondés ont déclaré que leurs exportations avaient augmenté et pour un quart d'entre eux de plus de $15 \% .85 \%$ s'attendent à une augmentation de leurs exportations en 2015 et $83 \%$ comptent développer de nouveaux produits dans un avenir proche. Irish Exporters Association, op. cit., p. 15.

71. $81,5 \%$ des sondés avaient confiance en l'avenir de leur entreprise en juin 2014. Un pourcentage qui n'était que de $62,5 \%$ en août 2013. 94,4\% pensaient que leurs revenus allaient augmenter et $87 \%$ s'attendaient à une hausse de leurs exportations. Irish Software Association, ISA Digital Technology Index, juin 2014. 
l'approche la plus appropriée? Quoi qu'il en soit, l'objectif de faire de l'Irlande le pays le plus propice à la création d'entreprise n’a pas encore été atteint, loin s'en faut. En témoigne le Global Entrepreneurship Index 2015 qui n’a classé le pays qu'au douzième rang des pays européens ${ }^{72}$.

72. Michael Hannigan, " Global Entrepreneurship 2015: Ireland in $12^{\text {th }}$ Rank in Europe, $17^{\text {th }}$ in World for Business Startups ", [http://www.finfacts.ielirishfinancenews/article_1028465.shtml], page consultée le 20 janvier 2015. 